Usages du français et pratiques d'enseignement en Europe balkanique, centrale et orientale - Grèce,

Serbie, Bulgarie, Moldavie, Hongrie, Allemagne, Russie - XVIIIe - XXe siècles

\title{
Enseigner le français en Russie au milieu du XVIIIe siècle. Pierre de Laval, précepteur et auteur d'une grammaire pour les Russes
}

\section{Sergueï Vlassov}

\section{(2) OpenEdition}

\section{Édition électronique}

URL : https://journals.openedition.org/dhfles/4145

DOI : $10.4000 /$ dhfles. 4145

ISSN : 2221-4038

Éditeur

Société Internationale pour l'Histoire du Français Langue Étrangère ou Seconde

Édition imprimée

Date de publication : 1 juin 2015

ISSN : $0992-7654$

Référence électronique

Sergueï Vlassov, «Enseigner le français en Russie au milieu du XVIIle siècle. Pierre de Laval, précepteur et auteur d'une grammaire pour les Russes », Documents pour l'histoire du français langue étrangère ou seconde [En ligne], 54 | 2015, mis en ligne le 01 janvier 2018, consulté le 26 mars 2023. URL : http://journals.openedition.org/dhfles/4145; DOI : https://doi.org/10.4000/dhfles.4145

Ce document a été généré automatiquement le 26 mars 2023.

Tous droits réservés 


\title{
Enseigner le français en Russie au milieu du XVIIIe siècle. Pierre de Laval, précepteur et auteur d'une grammaire pour les Russes
}

\author{
Sergueï Vlassov
}

\section{Introduction ${ }^{1}$}

1 Les premières grammaires françaises pour les Russes sont publiées en Russie au début des années 1750, à l'époque de l'impératrice Élisabeth. La première en date (1752) est la grammaire de Restaut traduite de l'allemand par Vassili Teplov (1752), traducteur de l'Académie des sciences de Saint-Pétersbourg. La deuxième $\left(1753^{2}\right)$ est due à un certain «Mr. De Laval», précepteur français du jeune prince Iouri Troubetskoï, fils du procureur général du Sénat Nikita Troubetskoï. Cette première grammaire bilingue du français éditée en Russie a pour titre Explication de la Grammaire Françoise avec de nouvelles observations, et des exemples sensibles sur l'usage de toutes ses parties (SaintPétersbourg, Imprimerie de l'Académie des sciences, 1752). Elle est dédiée par Laval à son élève ${ }^{3}$. Seul le nom de l'auteur de cette grammaire était connu. Les recherches aux Archives de l'Académie des sciences de Saint-Pétersbourg nous ont permis de mettre en lumière les activités variées de ce curieux personnage qui reflètent bien la vie des précepteurs étrangers en Russie au milieu du XVIII ${ }^{\mathrm{e}}$ siècle.

\section{2. École particulière de Monsieur de Laval}

2 Dès 1757, en vertu de l'ordre de l'impératrice Élisabeth et du décret du Sénat ${ }^{4}$, tout précepteur étranger arrivant en Russie devait se présenter à un examen auprès de l'Académie des sciences à Saint-Pétersbourg ou auprès de l'université de Moscou (Biliarski 1865 : 344). Pierre «Delaval » est l'un des premiers précepteurs étrangers à 
passer cet examen à l'Académie des sciences, le 14 mai 1757. Laval a déclaré qu' « il avait ici, à Saint-Pétersbourg, dans un appartement qu'il avait loué, une école particulière dans laquelle il enseignait aux jeunes le français, l'histoire, la géographie et l'arithmétique ». Selon l'attestation, « on l'a examiné dans ces matières et on a trouvé qu'il était capable de les enseigner » (SPF ARAN, fonds 3, op. 9, d. 78, f. 16).

En janvier et mars de la même année, Laval passe une annonce dans le journal de SaintPétersbourg :

Monsieur de Laval avec sa femme ont l'intention d'accueillir chez eux les jeunes filles qui désirent apprendre le français, la géographie, l'histoire, le dessin et l'arithmétique : les personnes intéressées peuvent se mettre d'accord avec lui sur le prix rue Millionnaïa, en face de la maison de Son Excellence le Comte Tchernychev. (SPbV 1757, $\mathrm{n}^{\circ} 7,24$ janvier ; $\mathrm{n}^{\circ} 22,18$ mars)

Le pensionnat change plusieurs fois d'adresse, mais continue d'exister au moins jusqu'en $1759\left(\mathrm{SPbV}, 1757, \mathrm{n}^{\circ} 30,15\right.$ avril ; $\mathrm{n}^{\circ} 75,19$ septembre ; 1758, $\mathrm{n}^{\circ} 20,10$ mars ; $1759, \mathrm{n}^{\circ} 62,3$ août).

\section{Service de Laval à l'Académie des sciences de Saint-Pétersbourg}

5 En 1759, commence une nouvelle période dans la vie de Laval. Il demande à la Chancellerie de l'Académie des sciences de l'engager à un poste vacant de maître de français et cite sa grammaire éditée par l'Académie comme preuve de ses talents ${ }^{5}$. Laval est engagé au collège académique en remplacement d'Henri de Lavie, "régent » ou professeur de la « haute classe » depuis 1752, qui présente sa démission le 17 juin 1759 (SPF ARAN, fonds 3, op. 1, d. 244, fo 214, 220) ${ }^{6}$. En s'engageant au service de l'État, Laval prête serment en apposant sa signature : « Pierre Delaval ${ }^{7}$. C'est le seul document qui nous permette d'établir avec certitude le prénom de l'auteur de l'Explication de la Grammaire Françoise.

Le travail de régent au collège académique est trop pesant pour Laval. Son absentéisme fait l'objet d'un rapport de Moderach, inspecteur de l'école académique: depuis longtemps, Laval ne va plus en classe sous prétexte que sa femme est malade. De la part de la Chancellerie académique, Lomonossov rappelle Laval à l'ordre en menaçant de réduire ses appointements (Biliarski $1865: 465)^{8}$.

7 Mécontent de son traitement et ne pouvant pas obtenir une augmentation de 100 roubles, Laval supplie en février 1763 la direction de l'Académie de «vouloir bien lui accorder un congé honorable, tel qu'il compte avoir mérité pour les soins qu'il a eus à remplir ses devoirs depuis 1759 » (SPF ARAN, fonds 3, op. 1, d. 273, f० 208-211). Il ne reçoit son congé qu'en juillet 1763 (ibid., fo 216). Un nouveau maître de français est nommé en remplacement de Laval, Jean-Charles Charpentier (ibid., fo 225), auteur d'une grammaire de la langue russe basée sur celle de Lomonossov? ${ }^{9}$.

\section{Histoire de la publication de la grammaire de Laval}

8 La traduction en russe de la grammaire de Laval est accomplie par deux traducteurs de l'Académie. Vassili Teplov s'y attaque d'abord, mais, malade, il doit la confier en février 1752 à Sergueï Voltchkov. Le texte de Laval et sa traduction doivent paraître dans le 
même volume, avec les textes français et russe en regard; Teplov et Voltchkov sont donc dans un sens les coauteurs de cette grammaire.

L'affaire est urgente car l'employeur de Laval, le procureur général Troubetskoï, presse la Chancellerie académique d'achever la traduction (SPF ARAN, fonds 3, op. 1, d. 521, fo 103). Voltchkov s'en acquitte vite: le 14 mai 1752, Laval prie "humblement" d'ordonner de publier sa grammaire aux frais de l'Académie (SPF ARAN, fonds 3, op. 1, d. 165 , fo 115). Le jour même J. D. Schumacher, directeur de la Chancellerie de l'Académie des sciences, signe un ordre prescrivant d'«imprimer à l'usage de la jeunesse russienne mille deux cents exemplaires in-quarto de cette grammaire sur papier russe utilisé pour les Commentaires académiques et douze exemplaires sur papier étranger royal de dimension moyenne » (SHF ARAN, fonds 3, op. 1, d. 521, fo 143v ; fonds 3, op. 1, d. 165, fo 116).

Cependant un obstacle inattendu surgit en juillet 1752 :

Monsieur le Président de l'Académie, Son Excellence le Comte [Cyrille Razoumovski], en partant pour la Petite Russie, a bien voulu donner un ordre oral de n'imprimer sans son approbation aucune épître dédicatoire à qui qu'elle soit adressée. Comme Monsieur de Laval demande d'imprimer auprès de l'Académie sa grammaire française qui est vue et approuvée par le Professeur Strube et qu'il veut accompagner d'une épître dédicatoire à son élève le Prince Iouri Nikititch Troubetskoï, il a été décidé d'envoyer ladite dédicace à Son Excellence le Comte pour son approbation. (SPF ARAN, fonds 3, op. 1, d. 521, fo 297)

11 Mais le 11 mars 1753, cette « dédicace » n'est toujours pas approuvée par Razoumovski car elle s'est égarée sur les routes pour la Petite Russie d'où le comte dirigeait alors l'Académie des sciences. On décide donc d'« envoyer une seconde fois un rapport à ce sujet à Son Excellence le Comte avec la même épître dédicatoire et d'en attendre l'approbation » (SPF ARAN, fonds 3, op. 1, d. 165, fo 120 ; fonds 3, op. 1, d. 522, fo 141). Le 26 juin 1753, après avoir enfin reçu l'approbation de Razoumovski, l'Académie décide d'«imprimer autant d'exemplaires de la dédicace qu'on a fait d'exemplaires de la grammaire » (SPF ARAN, fonds 3, op. 1, d. 522, fo 242 ; fonds 3, inv. 1, d. 165, fo 125).

Cette histoire anecdotique est probablement liée à la publication de la Nouvelle grammaire française dans la traduction de Vassili Teplov, à l'Académie des sciences de Saint-Pétersbourg, en 1751-1752. Cette grammaire était déjà utilisée au collège de l'Académie. L'administration académique voulait sans doute retarder la publication de la grammaire de Laval : celle-ci ne lui convenait pas tout à fait, mais l'Académie était contrainte de l'imprimer sous la pression du procureur général Troubetskoï.

La grammaire de Laval a été achevée d'imprimer le 23 août 1753 et a été mise en vente en septembre 1753 au prix d'un rouble l'exemplaire. Sur les 1262 exemplaires imprimés, l'auteur ne reçoit, " en récompense de son travail », que 47 exemplaires au lieu des cent qui lui étaient promis (PFA RAN, fonds 3, inv. 1, d. 165, fo 116, 129-134) ${ }^{10}$.

\section{Place de la grammaire de Laval dans l'histoire des manuels d'apprentissage du français en Russie}

La grammaire concurrente de Teplov n'est pas une œuvre originale, mais une traduction de l'édition allemande de la Nouvelle et parfaite Grammaire Françoise de Pierre Restaut (1749), elle-même basée sur d'autres grammaires (celles de J.-R. Des Pepliers, C. Buffier, P. de La Touche). La grammaire de Laval, elle, est une explication abrégée des 
Principes généraux et raisonnés de la grammaire françoise de Restaut (Restaut 1730). Cependant, elle fournit une grande quantité d'exemples qui ne sont pas empruntés à Restaut, mais inventés par Laval qui les adapte à la réalité russe de l'époque.

Dans la préface, l'auteur explique pourquoi il a abrégé et simplifié le texte de Restaut :

Mr. Restaut, tres habile Grammairien, m'a été d'un grand secours dans la composition de cet Ouvrage, mais j'ai évité, autant que j'ai pu, les expressions philosophiques dont sa Grammaire est remplie : Expressions qui ne me paroissent pas convenir dans un Ouvrage dont l'étude est d'elle même assez difficile, particulierement pour les enfans. (Laval 1752, $\mathrm{f}^{\circ} \mathrm{b} 2 \mathrm{v}$ )

Restaut a composé lui-même une variante simplifiée et abrégée de sa grammaire pour enfants (Restaut 1732) expurgée des « expressions philosophiques». Cette variante fut à la base de la traduction manuscrite d'Ivan Gorlitski présentée à la Chancellerie académique en 1748. Elle fut approuvée par les professeurs Strube, Trediakovski et Lomonossov qui l'ont alors recommandée à la publication après quelques corrections (Materialy $1900: 643-644)^{11}$.

17 La grammaire de Restaut, tout comme celles de Des Pepliers (1689) et de La Touche (1696), faisait partie des grammaires françaises les plus populaires en Russie au XVIII siècle. L'Abrégé des principes de la Grammaire françoise de Restaut a été souvent réédité en Russie (Restaut 1771, 1771, 1789, 1799, 1812). La grammaire de Restaut traduite par Teplov a connu elle aussi plusieurs rééditions $(1752,1762,1777,1787$ et 1809). Le succès du manuel de Teplov s'explique par le fait qu'il réunit dans un seul livre tout ce qu'il y a de meilleur dans les grammaires de Restaut, Des Pepliers et de La Touche.

18 Si les ouvrages de Des Pepliers et de La Touche se rattachent encore au vieux paradigme des grammaires formelles du XVII ${ }^{e}$ siècle, le traité plus moderne de Restaut porte l'empreinte des idées philosophiques de la Grammaire générale et raisonnée de PortRoyal (1660). Ces idées ont été développées dans la grammaire de C. Buffier (1709) qui était, avec celle de Regnier-Desmarais (1706), une des principales sources de Restaut. Il l'avoue lui-même dans la Préface de ses Principes généraux et raisonnés de la grammaire françoise (Restaut 1749a: XIX). Or cet aspect philosophique et logique du traité grammatical de Restaut ne convient pas à Laval et à d'autres précepteurs qui enseignent à cette époque le français aux enfants. En l'excluant, Laval reste, dans le fond, sur les vieilles positions de la grammaire formelle et traditionnelle, dans la mouvance de l'Art de bien parler français de Pierre de La Touche qu'il a utilisé dans son travail.

19 Tout en critiquant La Touche pour avoir rattaché le "futur passé » (Restaut désigne ainsi le futur antérieur) au « conjonctif » (Subjonctif), et non pas à l'indicatif, comme l'a fait judicieusement Restaut, Laval emprunte quelque chose à La Touche, mais d'une façon dissimulée. Par exemple, on trouve une citation cachée de La Touche dans la préface de la grammaire de Laval. Quand le précepteur énumère les qualités de la langue française, qui serait la langue la plus parfaite du monde, il écrit :

Tout le Monde convient qu'elle [la langue françoise] a tous les avantages des autres langues, sans en avoir les imperfections. Elle est tout ensemble Mâle et Délicate ; Simple et Majestueuse ; Agréable, Energique et Riche. Elle est propre pour la Poesie, comme pour la Prose, propre pour l'histoire et pour le Roman; pour le Serieux et pour le Comique. Ce qui a contribué à lui donner tous ces avantages, sont les soins que l'on a pris de l'épurer et de l'enrichir. (Laval 1752, $\mathrm{f}^{\circ} \mathrm{b} 1 \mathrm{v}^{\circ}$ )

Dans la préface de sa grammaire, La Touche caractérise le français presque avec les mêmes mots, mais de façon plus développée. Avec une petite différence : à la suite de D. 
Bouhours dont il utilise les épithètes pour faire valoir les traits distinctifs du français (Bouhours 1673: 52-86) ${ }^{12}$, La Touche affirme la supériorité du français sur l'allemand, l'anglais, l'espagnol et l'italien :

La Langue Alemande est énergique, mais elle est dure ; L'Anglaise est copieuse, mais elle n'est point châtiée; l'Espagnole est grave et pompeuse, mais elle est trop enflée ; l'Italienne est mignarde, mais elle est molle et languissante. La Langue Françoise seule a tous les avantages de ces Langues, sans en avoir les imperfections. Elle est tout ensemble douce et forte, éxacte et abondante, simple et majestueuse, mâle et délicate. Elle est propre à toutes sortes de matiéres, pour la prose et pour la poësie ; pour l'Histoire et pour le Roman ; pour le sérieux et pour le comique. On ne doit donc pas s'étonner de ce qu'elle est si digne de la prééminence qu'on lui donne sur toutes les langues vivantes, si l'on considére [sic] quels soins on prend depuis longtemps à l'épurer et à l'embellir. (La Touche 1696, $\mathrm{f}^{\circ} 5 \mathrm{v}^{\circ}-6$ )

Sans doute sous l'influence directe de Bouhours et de La Touche, mais aussi sous l'influence de Laval, sorte de chaînon intermédiaire entre Lomonossov et ses prédécesseurs français, le savant russe a reporté ces caractéristiques du français sur le russe. Citons la célèbre lettre dédicatoire au grand-duc Paul dont Lomonossov a fait précéder sa Grammaire russe (1755) :

Charles Quint, empereur romain, disait que l'espagnol convient pour parler avec Dieu, le français, avec des amis, l'allemand, avec l'ennemi, l'italien, avec le sexe féminin. Mais s'il avait maîtrisé le russe, il aurait sans doute ajouté que celui-ci convient pour parler avec eux tous car il aurait trouvé en lui la magnificence de l'espagnol, la vivacité du français, la force de l'allemand, la douceur de l'italien, et de plus la richesse et la grande concision de représentation du grec et du latin ${ }^{13}$. (Lomonossov 1952 : 391)

Du point de vue de la théorie grammaticale, l'Explication de la Grammaire Françoise représente des notes abrégées de la grammaire complète de Restaut, le plus souvent sous forme de citations littérales. Laval reproduit le système des quatre articles français selon Restaut, dans lequel le précepteur fait entrer les prépositions à et $d e^{14}$; l'article "défini » le, la, les; l'article "d'unité »" un, une; et l'article "partitif indéterminé »" $d u$, de la, des. Laval reproduit, en suivant toujours Restaut, les paradigmes des déclinaisons nominales, dix temps de l'indicatif auxquels sont rapportés le conditionnel présent et le conditionnel passé, ce qui était nouveau pour l'époque. Il cite de nombreux exemples d'emploi des parties du discours qu'on ne trouve pas chez Restaut. C'est peut-être l'unique innovation de cet auteur.

\section{En quoi consiste la nouveauté de la grammaire de Laval?}

Une grande quantité d'exemples dans la grammaire de Laval, particulièrement dans l'explication des parties du discours invariables (adverbes, prépositions et conjonctions), s'explique par l'objectif pratique de faire apprendre la grammaire française dans un milieu non-francophone à l'aide de la méthode grammairetraduction. Les phrases de la langue parlée liées à des situations de la vie quotidienne servent souvent d'exemples. Pour faciliter la mémorisation des mots, Laval recourt à la répétition d'un même mot:

Vous avez précisément fait ce qu'il ne faloit pas.

Je lui ay dit de venir précisément à cette heure la.

Nous soupons précisément à neuf heures. (p. 493 ${ }^{17}$ ) 

donner au lecteur plusieurs variantes : en faisant la traduction en sens inverse, du russe en français, l'étudiant russe peut voir comment ces différentes structures peuvent être exprimées en français. Le traducteur désire peut-être montrer les richesses lexicales et syntaxiques du russe : dans cette langue la phrase française peut être rendue de façon plus littérale ou plus libre, conformément à l'usage et au "génie» du russe. Le 
traducteur illustre ainsi la richesse de moyens synonymiques dans la langue russe : elle peut utiliser des mots slavons ${ }^{18}$ et russes, des emprunts et des mots propres.

Cette copia dicendi est dans le goût de la Renaissance si différent du modèle rhétorique français. Depuis Malherbe, mais surtout à partir de la deuxième moitié du XVII siècle, celui-ci se distingue par la recherche du " mot juste ». En effet, le traducteur ne suit pas les règles de l'abbé Girard, auteur des Synonymes français, connues en Russie grâce à Vassili Trediakovski: selon Girard, la richesse d'une langue ne s'exprime pas par l'abondance numérique des mots, mais par la justesse des synonymes. Comme l'écrivait Trediakovski dans son Discours sur l'éloquence :

31 L'accumulation de synonymes qui servent à désigner la même chose sans lui ajouter rien de nouveau est une éloquence creuse et non une éloquence vraie et ferme ${ }^{19}$.

Le traducteur de la grammaire de Laval campe plutôt sur des positions qui annoncent celles de Lomonossov. Ce dernier, souhaitant faire une synthèse entre les parties slavonne et russe du lexique en intégrant les deux dans la langue normalisée, ne distinguait pas les synonymes selon leur sens, mais selon leur registre ${ }^{20}$.

Mais cette redondance chez Voltchkov concerne plus particulièrement les termes linguistiques. La partie russe de la grammaire de Laval est donc très intéressante du point de vue de l'histoire des idées linguistiques en Russie. Dans la traduction, les termes modernes empruntés aux langues occidentales côtoient une terminologie vieillie toujours en usage, mais aussi des néologismes qui seront oubliés par la suite. La terminologie linguistique est alors en voie de formation en Russie. La traduction d'ouvrages linguistiques et didactiques comme la grammaire de Laval est une étape importante dans l'évolution de cette terminologie. Une influence de la grammaire de Laval sur celle de Lomonossov bien au-delà de la préface de celle-ci est envisageable (cf. supra). Certains termes grammaticaux russes proposés pour la première fois par Voltchkov ${ }^{21}$ semblent avoir été repris par Lomonossov dans sa Grammaire russe. Sans doute Lomonossov n'était-il pas le premier à les employer ${ }^{22}$. Nous voyons aussi à quelle richesse de possibilités un traducteur et un linguiste russe sont confrontés à cette époque. Le traducteur de Laval prend le parti de ne pas choisir et de donner la liste la plus large possible des équivalents des termes linguistiques français ${ }^{23}$.

Le traducteur ne traduit pas exactement certaines phrases en procédant à une sorte d'autocensure. Par exemple, quand il est question de Dieu, le traducteur est visiblement gêné par les exemples donnés par Laval et veut prévenir la censure ecclésiastique. Ainsi Laval écrit: "Qu'est-ce que Dieu?». Le traducteur ne peut pas remplacer la forme interrogative par la forme affirmative, mais il ajoute des épithètes: «Коль ВЕлик и чудЕн Бог? ", c'est-à-dire « Dieu combien est-il grand et miraculeux ? ».

Certains ajouts semblent venir du besoin d'expliquer ce qui devait être évident pour le lecteur occidental. Par exemple, la phrase « Personne a-t-il raconté plus naïvement que la Fontaine? ? est traduite de cette façon : « НИКТО ЕзОПОВЫХ БАСЕН, ТАК ИЗРЯДНО НЕ ПИСАЛ, КАК ГОСПОДИН ДЕ ЛА ФОНТЕН », c'est-à-dire «Personne n'a si bien écrit les fables d'Esope que Monsieur de La Fontaine ». La précision du traducteur porte non seulement sur la source des sujets de La Fontaine (Esope), mais aussi sur le genre (fable), car le lecteur russe faisant ses premiers pas dans l'apprentissage du français pouvait ignorer les deux.

36 La grammaire de Laval est intéressante à plusieurs points de vue : elle témoigne de l'intérêt accru pour le français en Russie et permet de comprendre sur quels ouvrages 
didactiques un maître de français s'appuie à cette époque. Les exemples, abondants et variés, sont un témoignage vivant de la pratique didactique d'un précepteur français en Russie au milieu du XVIII siècle. Enfin, la traduction de cette grammaire en russe jette une lumière sur la formation de la terminologie linguistique en Russie, alors à ses débuts.

\section{Annexes}

[Pierre] De Laval, Explication de la Grammaire Françoise avec de nouvelles observations, et des exemples sensibles sur l'usage de toutes ses parties. Dediée à son Altesse le Prince George Troubetskoye par Mr. De Laval Son Precepteur, [SaintPétersbourg, Académie des sciences], 1752 [1753], pages non numérotées.

Les deux exemplaires de cette grammaire conservés à la Bibliothèque nationale de France sont sans doute les seuls qu'on trouve dans les bibliothèques occidentales. Celui de l'Arsenal a appartenu à Théodore (Fedor) Karjavine, célèbre écrivain et traducteur russe, diplômé de la Sorbonne, fin connaisseur de la langue française. Son nom est imprimé sur la reliure du livre. À l'intérieur, sur l'une des premières pages, avant la page de titre, se trouve cette inscription, sans doute de la main de Karjavine lui-même : " $\mathrm{N}^{\circ}$. il y à une bien meilleure Grammaire d'une datte posterieure a cellecy par MM. Charpentier ${ }^{24}$ professeur de Langue Françoise au Corps des cadets à Petersbourg ». On sait que Karjavine, à un moment de sa carrière, a brigué le poste de traducteur de la bibliothèque royale, peut-être cet exemplaire est-il entré dans cette collection à cette époque. Le deuxième exemplaire de la BNF ne comporte aucune note manuscrite.

\section{1. Épître dédicatoire}

«Au prince George ${ }^{25}$ fils de Monseigneur le prince Troubetskoy ${ }^{26}[$,] Conseiller privé actuel d'Etat, Procureur General, Premier-Major des Gardes, et Chevalier des Ordres de l'Empire de Russie.

Mon prince !

Comme il n'est pas possible de bien posseder une langue sans en connoitre les principes, c'est une necessité indispensable d'aprendre la Grammaire. Mais la pluparte [sic !] de celles qui ont paru jusqu'à present, étant chargées de regles multipliées sans necessité, sont d'une Etude difficile et qui rebute, c'est ce qui m'a déterminé à faire l'explication que j'ai l'honneur de Vous presenter aujourd'hui, où Vous trouverés, Mon Prince, une définition précise de toutes les regles de la Grammaire, et des exemples sensibles qui Vous en faciliteront l'intelligence et l'usage.

Ce n'est point pour m'eriger en Auteur que j'ai pris la peine de faire cet ouvrage, j'ai cherché à Vous en epargner, en Vous adoucissant cette Etude si necessaire, pour parvenir à la pureté d'une langue à laquelle Vous donnés Vos soins. Je n'ay fait en cela que ce qu'un Precepteur doit à son Eleve; mais pensés serieusement Mon Prince à ce que Vous devés à Vous même. Vous etes né Prince, sachés que cette qualité n'est respectable qu'autant qu'elle est soutenuë par le merite, sans quoi elle est bien peu de chose. Un homme de commun qui en a, n'est-il pas plus estimable que le premier homme de condition qui en est dépourvu ? à quoi ce dernier est-il propre ? le Souverain peut-il lui donner la conduite de ses armées, ou le mettre à la tete de la justice ? Cela 
n'est pas naturel, il le regarde comme un homme inutile, et lui donne tout au plus une charge honoraire, pendant que l'homme de merite sert utilement l'Etat, et en est un des premiers.

Oui, Mon Prince, la naissance n'est rien, si elle n'est acompagnée d'un merite qui met en Etat de servir avec distinction son Souverain et sa Patrie. Comment aquiert on ce merite? C'est par l'etude, c'est elle qui fait les grands hommes que la nature ne fait qu'ebaucher. Vous ne pouvés mieux faire que de Vous appliquer à la langue française, dans laquelle Vous trouverés tant d'Auteurs respectables, qui ne laissent rien à desirer à l'homme qui veut s'eclairer, se perfectionner le cœur et l'esprit. Travaillés donc, Mon Prince, à être bientôt à même de Vous entretenir avec eux[,] l'Etude est du gout de toutes les personnes raisonnables; C'est la plus belle et la plus noble ocupation de l'homme; C'est un exercice purement spirituel, où le corps a beaucoup moins de part que l'Esprit. On ne peut devenir savant sans le secours de l'Etude qui nous polit et nous fait raisonner juste. C'est par l'Etude que nous aprennons quel est l'excellent homme de guerre, le sage Legislateur, le juge equitable, le bon Citoyen, en un mot l'homme de merite. Quel plaisir enfin n'aurés Vous pas, Mon Prince, de remarquer dans l'Histoire, les diferentes revolutions qui se sont faites dans le Monde, d'y trouver des modeles de vertus à suivre, et des vices à eviter.

Je m'estimerai fort heureux, Mon Prince, si Vous profités des avis que je Vous donne, Vous en aurés la gloire et l'avantage qu'il en revient toujours de faire le bien, et moi la satisfaction d'avoir contribué à l'une et à l'autre.

J'ai l'honneur d'être

MON PRINCE

Vôtre tres humble et tres obeissant Serviteur

DE LAVAL »

\section{Préface}

Un Auteur ne se borne pas ordinairement dans une Preface, à rendre compte de la conduite qu'il a tenuë dans l'Ouvrage qu'il donne au public, il fait aussi l'eloge du sujet de son Ouvrage, ainsi je devrois faire ici celui de la Langue Française, si le soin que toutes les nations prennent à l'apprendre, n'en etoit pas un plus beau que celui que je pourrois faire. Tout le Monde convient qu'elle a tous les avantages des autres langues, sans en avoir les imperfections. Elle est tout ensemble Mâle et Délicate; Simple et Majestueuse ; Agréable, Energique et Riche. Elle est propre pour la Poesie, comme pour la Prose, propre pour l'Histoire et pour le Comique. Ce qui a contribué à lui donner tous ces avantages, sont les soins que l'on a pris de l'epurer et de l'enrichir.

Pour rendre compte de cet Ouvrage, je me contenterai de dire que la Grammaire étant un composé de Regles, on ne peut en donner une Explication ni trop précise, ni trop simple, sans quoi l'Etude en est désagréable et rebutante aux jeunes gens. Plusieurs Grammairiens ont été trop laconiques; d'autres ont été trop difus, en entassant regles sur les regles; mon attention a été de ne point tomber dans ces deux extremités. J'explique la Grammaire avec toute la précision qu'il m'a été possible, et je donne des Exemples qui ne laissent rien à desirer sur l'usage de toutes les parties. 
51 Mr. Restaut, tres habile Grammairien, m'a eté d'un grand secours dans la composition de cet Ouvrage, mais j'ai evité, autant que j'ai pû, les expressions philosophiques dont sa Grammaire est remplie: Expressions qui ne me paroissent pas convenir dans un Ouvrage dont l'etude est d'elle-même assez difficile, particulierement pour les enfans. Mr. Restaut s'est d'ailleurs trompé au sujet du second Cas, voici comment. Le Genitif exprime, comme il le dit, le raport d'une chose qui apartient à une autre, mais il paroit y avoir de l'inatention dans les exemples qu'il donne de ces raports, ainsi qu'on en peut juger par celui-ci, et plus amplement par l'examen de cette partie de sa Grammaire et de cet Ouvrage. qu'un mois est une partie de l'année et que la porte est une partie de la maison? qu'ainsi le titre du tout à la partie n'est pas juste, et qu'il faudroit mettre de la partie au tout.

Je finirai cette Preface, en disant que quelqu'un poura trouver à redire que je ne me suis pas assez étendu sur les Voyelles, les Consonnes et les Diphtongues, mais j'avertis que je l'ay fait à dessein, ce qu'on en dit de plus me paroissant fort inutile. A l'egard de l'arangement des parties de cet Ouvrage, j'ai été attentif à le faire suivant la liaison qu'elles ont entre elles, et en conduisant mon eleve comme de classe en classe.

Je préviens le public que cette explication de la Grammaire n'a été faite que pour mes eleves, mais m'ayant été representé qu'elle pouvoit être de quelques utilité à la Jeunesse Russienne qui aprend la Langue Française, je me suis volontiers déterminé à la lui donner.

\section{BIBLIOGRAPHIE}

\section{Sources primaires}

\section{Documents d'archives}

SPF ARAN (Archives de l'Académie des sciences de Russie, Saint- Pétersbourg), fonds 3, op. 1, d. 165.

SPF ARAN, fonds 3, op. 1, d. 244.

SPF ARAN fonds 3, op. 1, d. 246.

SPF ARAN, fonds 3, op. 1, d. 521.

Documents pour l'histoire du français langue étrangère ou seconde, 54 | 2015 
SPF ARAN, fonds 3, op. 1, d. 522.

SPF ARAN, fonds 3, op. 9, d. 78.

\section{Sources imprimées}

[ARNAULD, Antoine \& LANCELOT, Claude] (1660). Grammaire Generale et Raisonnée. Paris : P. Le Petit.

BARANOV, Platon (1875). ОПИСЬ ВЫСОЧАЙШИМ УКАЗАМ И ПОВЕЛЕНИЯМ, ХРАНЯЩИМСЯ В САНКТПЕТЕРБУРГСКОМ СЕНАТСКОМ АРХИВЕ, ЗА ХVIII ВЕК. Т. 3, САНКТ-ПЕТЕРБУРГ [Platon Baranov, Index des oukases et décrets impériaux conservés aux Archives du Sénat à Saint-Pétersbourg. Vol. 3. SaintPétersbourg].

[BOUHOURS, Dominique] (1673) [1671]. Les Entretiens d'Ariste et d'Eugene. Paris : S. MabreCramoisy.

BUFFIER, Claude (1709). Grammaire Françoise sur un plan nouveau. Paris : N. Le Clerc, M. Brunet, Leconte et Montalant.

[CHARPENTIER, Jean-Baptiste Jude, MARIGNAN] (1768). Elements de la langue russe ou Methode courte et facile pour apprendre cette langue conformément à l'usage. Saint-Pétersbourg : de l'imprimerie de l'Académie des sciences [16]. 368 pages, 1 tabl. (Rééditions : Saint-Pétersbourg, $1791,1795,1805)$.

GRAMMAIRE Françoise et Russe en Langue moderne (1730). Grammaire Françoise et Russe en Langue moderne accompagnée d'un petit Dictionnaire pour la Facilité du commerce / ГРАММАТИКА ФРАНЦУСКАЯ И РУССКАЯ НЫНЊШНЯГО ЯЗЫКА СООБЩЕНА СЪ МАЛЫМЪ ЛЕКСИКОНОМЪ РАДИ УДОБНОСТИ СООБЩЕСТВА. Saint-Pétersbourg : [Imprimerie de l'Académie des sciences].

[LA TOUCHE, Pierre de] (1696). L'Art de bien parler François. Vol. 1-2. Amsterdam : Henri Desbordes (plusieurs rééditions).

LAVAL, [Pierre] de (1752-1753). Explication de la Grammaire Françoise avec de nouvelles observations, et des exemples sensibles sur l'usage de toutes ses parties. Dediée à son Altesse le Prince George Troubetskoye par Mr. De Laval Son Precepteur. ИЗЪЯСНЕНИЕ НОВОЙ ФРАНЦУССКОЙ ГРАММАТИКИ С ПРИМЕЧАНИЯМИ И ПРИМЕРАМИ НА ВСЕ ЧАСТИ СЛОВА. St.-Pétersbourg : pri Imperatorskoї Akademii naouk.

LOMONOSSOV, Mikhaïl (1952). ПОЛНОЕ СОБРАНИЕ СОЧИНЕНИЙ [๕uvres complètes]. Vol. VII. MoscouLeningrad : Éditions de l'Académie des sciences de l'URSS.

MATERIALY (1900). МАТЕРИАЛЫ ДЛЯ ИСТОРИИ ИМПЕРАТОРСКОЙ АКАДЕМИИ НАУК [Documents pour l'histoire de l'Académie impériale des sciences]. Vol. X. Saint-Pétersbourg : Imprimerie de l'Académie des sciences.

MAUPAS, Charles (1625) [1607]. Grammaire et Syntaxe Françoise. Seconde Edition. ReueuË, corrigee, et augmentee de moitié, et en beaucoup de sortes amendee, outre la precedente, par ledit Autheur. Paris : Adrian Bacot.

MONTAIGNE, Michel (1762). миХАЙлА монтАниЕвыЕ опыты [Les Essais de Michel de Montaigne]. Saint-Pétersbourg : Imprimerie du Sénat.

PEPLIERS, Jean-Robert Des (1689). Grammaire Royale Françoise et Allemande, contenant une Methode nouvelle et façile [sic !] pour apprendre en peu de temps la langue françoise, Avec une Nomenclature, des Dialogues nouveaux, Bouquet des Sentences, des Lettres et billets galants de ce temps, ecrite par Mr Jean Robert des Pêpliers, Informateur de Monseig. Le Duc de Bourgogne [...] Berlin : Johan Völcker (plusieurs 
rééditions au XVIII ${ }^{\mathrm{e}}$ siècle, considérablement refondues. Il y a une traduction russe de F. Sokolski, Moscou, 1780).

REGNIER-DESMARAIS, François-Séraphin (1706). Traité de la Grammaire Françoise. Paris : J.-B. Coignard.

[RENAUD, Antoine] (1697). Maniere de parler la langue françoise selon les differens styles. Lyon : C. Rey.

RESTAUT, Pierre (1730). Principes généraux et raisonnés de la grammaire françoise. Paris : J. Desaint (plusieurs rééditions 1732, 1736, 1741, 1745, 1749, 1750, etc.).

RESTAUT, Pierre (1732). Abrégé des principes de la Grammaire Françoise. Paris : J. Desaint.

RESTAUT, Pierre (1749a). Principes généraux et raisonnés de la Grammaire Françoise. $5^{\mathrm{e}}$ éd. Paris : Ph. N. Lottin.

RESTAUT, Pierre (1749b). Nouvelle et parfaite Grammaire Françoise. Neue und vollstaendige Franzoesische Grammatic, in Frag und Antwort abgefasset. Aus dem Franzoesischen des Herrn Restaut und andern Anmerkungen der besten Franzoesischen Sprachlehrer zusammen getragen. Mainz und Frankfurt am Mayn, bei Franz Warrentrapp.

RESTAUT, Pierre (1752). НОВАЯ ФРАНЦУССКАЯ ГРАММАТИКА СОЧИНЕННАЯ ВОПРОСАМИ И ОТВЊТАМИ. СОБРАНА ИЗЪ СОЧИНЕНИЙ ГОСПОДИНА РЕСТО И ДРУГИХЪ ГРАММАТИКЪ, А НА РОССИЙСКОЙ ЯЗЫКЪ ПЕРЕВЕДЕНА АКАДЕМЇИ НАУКЪ ПЕРЕВОДЧИКОМЪ ВАСИЛЬЕМЪ ТЕПЛОВЫМЪ, ВЪ САНКТПЕТЕРБУРГЬ ПРИ ИМПЕРАТОРСКОЙ АКАДЕМЇИ НАУКЪ [La nouvelle grammaire française par demandes et réponses. Recueillie des ouvrages de Monsieur Restaut et d'autres grammaires et traduite en russe par le traducteur de l'Académie des Sciences Vassiliï Teplov]. Saint-Pétersbourg, auprès de l'Académie impériale des Sciences]. Les éditions ultérieures (Saint-Pétersbourg, 1762, 1777, 1787, 1809) portent le titre ФРАНЦУЗСКАЯ ГРАММАТИКА СОБРАННАЯ ИЗЪ РАЗНЫХЪ АВТОРОВЪ ГОСПОДИНОМЪ РЕСТО, A НА РОССІЙСКОЙ ЯЗЫКЪ ПЕРЕВЕДЕННАЯ ВАСИЛЬЕМЪ ТЕПЛОВЫМЪ [Grammaire française recueillie de différents auteurs par Restaut et traduite en russe par Vassili Teplov].

RESTAUT, Pierre (1771). Abrégé des principes de la Grammaire Françoise. Saint-Pétersbourg : Imprimerie du Corps des cadets de terre, 1771 (rééditions en 1789 et en 1799).

RESTAUT, Pierre (1812). СОКРАЩЕННЫЕ ПРАВИЛА ФРАНЦУЗСКОЙ ГРАММАТИКИ [Abrégé des principes de la grammaire française], Saint-Pétersbourg, 1812. Cette édition a paru avec une traduction russe en regard faite par A. E. Danilovski.

SPbV (1757) - САНКТ-ПЕТЕРБУРГСКИЕ ВЕДОМОСТИ [Journal de Saint-Pétersbourg].

VOLTCHKOV, SergueÏ (1755). НОВЫЙ ЛЕКСИКОН НА ФРАНЦУССКОМЪ, НЊМЕЦКОМЪ, ЛАТИНСКОМЪ, И НА РОССИЙСКОМЪ ЯЗЫКАХЪ, ПЕРЕВОДУ АСЕССОРА СЕРГЬЯ ВОЛЧКОВА. ЧАСТЬ ПЕРЬВАЯ СЪ ЛИТЕРЫ А, ПО лИТЕРУ $G$ [Nouveau dictionnaire français, allemand, latin et russe, traduit par l'assesseur Voltchkov. Première partie de la lettre A à la lettre G]. Saint-Pétersbourg: Imprimerie de l'Académie des sciences, s.a.

VOLTCHKOV, SergueÏ (1764). НОВАГО ВОЯЖИРОВА ЛЕКСИКОНА НА ФРАНЦУССКОМ, НЊМЕЦКОМЪ, ЛАТИНСКОМъ, И НА РОССИЙСКОМЪ ЯЗЫКАХЪ, ЧАСТЬ ВТОРАЯ СЪ ЛИТЕРЫ G, ДО КОНЦА АЛФАВИТА [Seconde partie du Nouveau dictionnaire du voyageur français, allemand, latin et russe, de la lettre G jusqu'à la fin de l'alphabet]. Saint-Pétersbourg : Imprimerie de l'Académie des sciences.

\section{Sources secondaires}

ARCHAIMBAULT, Sylvie (1999). «Les Élémens de la langue russe de J.-B. J. Charpentier ou les Français et l'apprentissage de la langue russe au XVIII ${ }^{\mathrm{e}}$ siècle ». In Gerda Hassler \& Peter 
Schmitter (dir.). Sprachdiskussion und Beschreibung von Sprachen im 17 und 18. Jahrhundert. Münster : Nodus Publikationen, 371-380.

BILIARSKI, Piotr (1865). МАТЕРИАЛЫ ДЛЯ БИОГРАФИИ ЛОМОНОСОВА. САНКТ-ПЕТЕРБУРГ, ТИПОГРАФИЯ АКАДЕМИИ НАУК [Petr Biliarski. Documents pour la biographie de Lomonossov. Saint-Pétersbourg : Imprimerie de l'Académie des sciences].

BOULITCH, Sergueï (1904). очЕРК ИстоРИИ ЯЗЫКОЗНАНИЯ В РОсСиИ [Аperçu de l'histoire de la linguistique en Russie]. Vol. I (XIII ${ }^{\mathrm{e}}$ s. - 1825). St.-Pétersbourg : Tipografiia M. Merkoucheva. BREUILLARD, Jean (2006). «Les synonymes de l'Abbé Girard en Russie ». Dix-huitième siècle. $2006 / 1, n^{\circ} 38,195-223$.

KAREVA, Natalia (2011). ФОРМИРОВАНИЕ ТЕРМИНОЛОГИИ ГУМАНИТАРНЫХ НАУК : НАИМЕНОВАНИЯ ГЛАГОЛЬНЫХ КАТЕГОРИЙ В « РОССИЙСКОЙ ГРАММАТИКЕ » М.В. ЛОМОНОСОВА (1755). СТАНОВЛЕНИЕ ПЕТЕРБУРГСКОЙ АКАДЕМИЧЕСКОЙ ФИЛОЛОГИЧЕСКОЙ ТРАДИЦИИ [Formation de la terminologie des sciences humaines : dénominations des catégories verbales dans la ‘Grammaire russe' de M.V. Lomonossov (1755). Les origines de la tradition philologique académique de Saint-Pétersbourg]. Thèse. Saint-Pétersbourg : Institut de recherches linguistiques. En ligne : <http://lomonosov. iling.spb.ru/>.

VLASSOV, SergueÏ \& MOSKOVKINE, Léonide (2007). « ИЗ ИСТОРИИ СОЗДАНИЯ УчЕБНИКОВ РУССКОГО ЯЗЫКА КАК ИНОСТРАННОГО В РОССИИ : ‘ОСНОВЫ РУССКОГО ЯЗЫКА' ШАРПАНТЬЕ И МАРИНЬЯНА (1768) ». МИР РУСскОГО сЛОВА [« De l'histoire des manuels de russe comme langue étrangère en Russie, 'Les élémens de la langue russe...' (1768) ». Le Monde de la parole russe, revue]. $n^{\circ} 1 / 2,72-80$.

VLASSOV, SergueÏ \& MOSKOVKINE, Léonide (2008). « ИЗ ИСТОРИИ СОЗДАНИЯ УЧЕБНИКОВ РУССКОГО ЯЗЫКА КАК ИНОСТРАННОГО В РОССИИ : 'ГРАММАТИКА ФРАНЦУЗСКАЯ И РУССКАЯ ...' (1730 год) », миР РУсского словА [ « De l'histoire des manuels de russe comme langue étrangère en Russie. 'La Grammaire françoise et russe...' (1730) », Le Monde de la parole russe, revue], ${ }^{\circ} 2$, $82-90$.

VLASSOV, SergueÏ \& RJEOUTSKI, Vladislav (2011). « Charpentier, Jean-Baptiste Jude ». In Anne Mezin \& Vladislav Rjeoutski (dir.), Les Français en Russie au siècle des Lumières. Ferney : CIEDS. Vol. $2,169-171$.

VLASSOV, Sergueï (2011). «V. K. Trediakovskij et les théories françaises du bon usage ». Revue des études slaves, t. 82/2, 217-251.

VLASSOV, SergueÏ \& MOSKOVKINE, Léonide (2013). « НЕКОТОРЫЕ НОВЫЕ СООБРАЖЕНИЯ ПО ПОВОДУ ИСТОРИИ СОЗДАНИЯ « ГРАММАТИКИ ФРАНЦУЗСКОЙ И РУСКОЙ ... » 1730 ГОДА, ВЕСТНИК СПБГУ, СЕРИЯ 9, ВЫПУСК 3, 125-137 [Quelques nouvelles considerations sur l'histoire de la rédaction de 'La Grammaire françoise et russe...' (1730) ». Messager de l'Université d'État de Saint-Pétersbourg. Série 9. fasc. 3, 125-137].

\section{NOTES}

1. Avec la collaboration de Vladislav Rjéoutski qui a contribué à la partie sur la traduction de la grammaire de Laval et a saisi les textes publiés.

2. Malgré la date de 1752 indiquée sur la page de titre.

3. La Grammaire Françoise et Russe en Langue moderne accompagnée d'un petit Dictionnaire pour la Facilité du commerce / ГРАММАТИКА ФРАНЦУСКАЯ И РУССКАЯ НЫНњШНЯГО ЯЗЫКА 
СООБЩЕНА СЪ МАЛЫМЪ ЛЕКСИКОНОМЪ РАДИ УДОБНОСТИ СООБЩЕСТВА, Saint-Pétersbourg, 1730, qui est considérée parfois comme la première grammaire du français publiée en Russie, est en fait un manuel d'apprentissage du russe pour les étrangers et non pas une grammaire française. Voyez pour plus de détails : Sergueï Vlassov \& Léonide Moskovkine, 2011, 2013.

4. L'oukase du 29 avril 1757 et le décret en date du 5 mai 1757 (Baranov 1875 : 342).

5. La demande est datée du 20 août 1759 (SPF ARAN fonds 3, op. 1, d. 246, fo 331).

6. Laval entre en fonctions le 17 septembre 1759, avec un traitement de 300 roubles par an (SPF ARAN, fonds 3, op. 1, d. 246, fo 336 -337).

7. Le texte du serment est en allemand (ibid., $\mathrm{f}^{\circ} 335$ ).

8. Le rapport de Moderach est daté du 19 octobre 1760.

9. Écrite en collaboration avec un autre enseignant français, Marignan (Charpentier, Marignan 1768). Voir sur Charpentier (Vlassov, Moskovkine 2007).

10. L'impression a coûté à l'imprimerie académique 725 roubles 89 kopecks.

11. Cependant en juin 1750 le traducteur Vassili Teplov présente à l'Académie sa traduction de l'édition allemande de la grammaire de Restaut approuvée aussi par Trediakovski et Lomonossov qui ont pris en novembre 1750 la décision de la publier en y ajoutant les dialogues de la grammaire de Gorlitski; pour celui-ci, on a décidé de publier une autre grammaire qu'il a traduite et qui s'appelait Les principes de la langue française. Cependant cette dernière n'a pas été publiée non plus, probablement parce qu'on a décidé de publier aux frais de l'Académie, outre la grammaire de Restaut traduite par Teplov, celle de Laval.

12. Cf. Renaud $1697: 15-44$.

13. Traduit du russe: «КАРЛ ПЯТЫЙ, РИМСКИЙ ИМПЕРАТОР, ГОВАРИВАЛ, ЧТО ИШПАНСКИМ ЯЗЫ์КОМ С БОГОМ, ФРАНЦУЗСКИМ - С ДРУЗЬЯМИ, НЕМЕЦКИМ - С НЕПРИЯТЕЛЬМИ, ИТАЛИЯНСКИМ - С ЖЕНСКИМ ПОЛОМ ГОВОРИТЬ ПРИЛИЧНО. НО ЕСЛИ БЫ ОН РОССИЙСКОМУ ЯЗЫ́КУ БЫЛ ИСКУСЕН, ТО, КОНЕЧНО, К ТОМУ ПРИСОВОКУПИЛ БЫ, ЧТО ИМ СО ВСЕМИ ОНЫМИ ГОВОРИТЬ ПРИСТОЙНО, ИБО НАШЕЛ БЫ В НЕМ ВЕЛИКОЛЕПИЕ ИШПАНСКОГО, ЖИВОСТЬ ФРАНЦУЗСКОГО, КРЕПОСТЬ НЕМЕЦКОГО, НЕЖНОСТЬ ИТАЛИЯНСКОГО, СВЕРХ ТОГО БОГАТСТВО И СИЛЬНУЮ В ИЗОБРАЖЕНИЯХ КРАТКОСТЬ ГРЕЧЕСКОГО И ЛАТИНСКОГО ЯЗЫ́КА » (ibid.).

14. Que la tradition grammaticale remontant au XVIIe siècle considérait comme des articles « indéfinis ».

15. Terme remontant à la grammaire de Maupas (1607).

16. Terme de Buffier remontant aussi à Maupas.

17. Nous indiquons dorénavant dans le corps du texte la page correspondante de la grammaire de Laval.

18. Le slavon était la langue de l'Église et la langue de l'écriture en Russie avant le XVIIIe siècle. On assiste donc à cette époque à une sorte de diglossie quand le russe parlé s'introduit dans le domaine de l'écriture littéraire.

19. Cité d'après Jean Breuillard (2006) et dans sa traduction, ici p. 207. Cf. aussi Sergueï Vlassov (2011 : 225-226).

20. Voir au sujet de la conception de Lomonossov Jean Breuillard (2006:211).

21. Tels que « ВСПОМОГАТЕЛЬНЫЙ ГЛАГОЛ » et « ВЗАИмНЫЙ ГЛАГОЛ ».

22. Contrairement à l'avis de Natalia Kareva (2011 : 77-82, 114-116, 230-23).

23. Voici quelques exemples de termes modernes: ПроизношЕнїЕ, Имя, МњстоимЕнїЕ, ГЛАГОЛЪ, ПРИЧАСТЇЕ, НАРњЧЇЕ, ПРЕДЛОГЪ, СОЮЗЪ, МЕЖДОМЕТЇЕ; ЛИЧНЫЯ, ПРИТЯЖАТЕЛЬНЫЯ, УКАЗАТЕЛЬНЫЯ И НЕОПРЕДњЛЕННЫЯ МЬСТОИМЕН̈̈Я. On rencontre à côté de ceux-là les termes vieillis mais toujours utilisés au milieu du XVIIIe siècle en Russie comme ЗВонъ (son), остРАЯ / ТЯЖКАЯ сИЛЫ (accent aigu / accent grave), ЧАстИ словА (parties du discours), ВОПРОСИТЕЛЬНАЯ (point d'interrogation), УДИВИТЕЛЬНАЯ (point d'exclamation), ВЛАДњТЕЛЬНЫЯ МњСТОИМЕНїЯ (pronoms possessifs), ПРЕХОДящЕЕ (passé imparfait), 
ДњЕГЛАГОЛїЕ (gérondif), ПРИСЛОВїЕ / ПРИРњЧїЕ (adverbe), etc. Enfin, on y voit plusieurs néologismes qui ne prendront pas racine dans la tradition linguistique russe, parmi lesquels des calques et des emprunts directs: ЧАСТИ РАЗГОВОРА (parties du discours), ЧЛЕНОВНЫЕ ЗВОНЫ (sons distincts), ЛИТЕРА (lettre), é ЗАМКНУтоЕ (é fermé), ПРОНУНЦїАЦ̈̈Я / ВЫГОВАРИВАНЇЕ (prononciation), УЧАСТНЫЙ ЧЛЕН (article partitif), МњСТОИМЕНЇЯ СХОДСТВЕННЫЯ (pronoms relatifs), ПРОНОМИНЫ (pronoms), ОКОНЧАТЕЛЬНЫЕ / НЕОКОНЧАТЕЛЬНЫЕ ЧЛЕНЫ, ВРЕМЕНА (articles / temps définis et indéfinis), оБРАЗЫ / кЛАсСЫ (modes), СПРЯГАТЕЛЬНЫЙ ОБРАЗЪ (mode conjonctif, ou subjonctif), ПРОШЕДШЕЕ ДАВНњШНЕЕ / ДАВНО СОВЕРШЕННОЕ (plus-queparfait), УЧАСТїЕ (adverbe), АДВЕРБъ (adverbe), etc.

24. Il s'agit de Jean-Baptiste Jude Charpentier, voir sur lui Sylvie Archaimbault (1999), Sergueï Vlassov et de Vladislav Rjéoutski (2011); et l'article cité de Sergueï Vlassov et Léonide Moskovkine (2007).

25. Le prince Iouri Nikititch Troubetskoï (1736-1811), futur conseiller privé actuel, franc-maçon, ami du célèbre franc-maçon et éditeur russe Nikolaï Novikov. L'élève de Laval avait dix-sept ans au moment de la publication de sa grammaire.

26. Le prince Nikita Iourievitch Troubetskoï (1699-1767), conseiller privé actuel, général-feldmaréchal, procureur général du Sénat.

\section{RÉSUMÉS}

Dans cet article nous nous proposons d'analyser de nombreuses activités pédagogiques de Pierre Laval, maître de français et auteur d'une première grammaire française pour les Russes publiée en français à Saint-Pétersbourg, au début des années 1950 du XVIIIe siècle. Laval, tout en suivant sa source principale, la grammaire de Restaut, complète son modèle par des exemples « sensibles » et originaux liés à des situations de la vie quotidienne et adaptés à la réalité russe de l'époque. Le traité de Laval est accompagné d'une traduction russe en regard qui a contribué à l'élaboration de la terminologie grammaticale russe. L'article est suivi de deux annexes, de l'épître dédicatoire au prince Youri Troubetskoï et de la Préface dans lesquelles Laval expose ses idées sur l'éducation en général et sur l'enseignement de la grammaire française en particulier.

The article analyzes many educational activities of Pierre Laval, French language teacher and author of the first French grammar for Russians published in French in St. Petersburg, in the early 50 s of the 18th century. Laval, while following his main source, Restaut's grammar, completes its model by "sensitive" and original examples associated with situations of everyday life and adapted to Russian realities of the time. The Laval treaty is accompanied by a Russian translation that contributes to the development of Russian grammatical terminology. The article is followed by two appendices, the dedication to Prince Yuri Trubetskoy and the Preface in which Laval exposes his ideas on education in general and on the teaching of French grammar in particular. 
INDEX

Mots-clés : Pierre de Laval, enseignement du français, grammaires françaises pour les Russes, terminologie grammaticale, Pierre Restaut, Sergueï Voltchkov, Mikhaill Lomonossov.

Keywords : Pierre Laval, teaching French, French grammars for Russians, grammatical terminology, Pierre Restaut, Sergei Voltchkov, Mikhail Lomonossov.

\section{AUTEUR}

\section{SERGUEII VLASSOV}

Université de Saint-Pétersbourg 